\title{
Affordable membrane permeability calculations: Permeation of short-chain alcohols through pure-lipid bilayers and a mammalian cell membrane. Supporting Information
}

Chi Hang Tse,${ }^{\dagger}$ Jeffrey Comer,,${ }^{\ddagger}$ Simon Kit Sang Chu,${ }^{\dagger}$ Yi Wang $^{,},{ }^{, \uparrow}, \dagger$ and Christophe Chipot*, $, \S, \|$

$\dagger$ †epartment of Physics, The Chinese University of Hong Kong, Shatin, Hong Kong SAR, China $\ddagger$ Institute of Computational Comparative Medicine and Nanotechnology Innovation Center of Kansas State, Department of Anatomy and Physiology, Kansas State University, Manhattan, Kansas 66506

IShenzhen Research Institute, The Chinese University of Hong Kong, Shatin, Hong Kong SAR, China

§Laboratoire International Associé Centre National de la Recherche Scientifique et University of Illinois at Urbana-Champaign, Unité Mixte de Recherche n7019, Université de Lorraine, B.P. 70239, 54506 Vandouvre-lès-Nancy cedex, France

||Beckman Institute for Advanced Science and Technology, University of Illinois at Urbana-Champaign, 405 North Mathews Avenue, Urbana, Illinois 61801

$\perp$ Department of Physics, University of Illinois at Urbana-Champaign, 1110 West Green Street, Urbana, Illinois 61801

E-mail: yiwang@cuhk.edu.hk; chipot@ks.uiuc.edu 
Table S1: Composition of the mammalian membrane model simulated in this work. The membrane contains altogether 26 types of components, with 127 and 129 molecules in its upper and lower leaflet, respectively. Lipid names correspond to residue names used by the CHARMM36 force field.

\begin{tabular}{l|c|c}
\hline Lipid & No. (upper leaflet) & No. (lower leaflet) \\
\hline ASM & 1 & 2 \\
CER241 & 0 & 1 \\
CHL1 & 38 & 43 \\
DAPE & 6 & 1 \\
DOPC & 1 & 1 \\
DOPE & 3 & 1 \\
LSM & 2 & 3 \\
NSM & 6 & 12 \\
OSM & 0 & 1 \\
PLPC & 12 & 26 \\
PLPE & 7 & 2 \\
PLPS & 2 & 0 \\
POPA & 1 & 0 \\
POPC & 8 & 17 \\
POPE & 8 & 2 \\
POPI14 & 1 & 0 \\
POPI2A & 1 & 0 \\
POPI33 & 1 & 0 \\
POPS & 3 & 0 \\
PSM & 4 & 9 \\
SAPC & 2 & 4 \\
SAPE & 7 & 2 \\
SAPS & 7 & 0 \\
SDPC & 0 & 1 \\
SDPE & 3 & 0 \\
SDPS & 3 &
\end{tabular}

Table S2: Equilibration time of systems initiated from different snapshots of the SMD trajectory. $z$ refers to the Euclidean distance between center of mass of the permeant to the center of a lipid bilayer projected along the membrane normal.

\begin{tabular}{r|r}
\hline$z(\AA)$ & Equilibration time (ns) \\
\hline \pm 35 & 10 \\
\pm 30 & 25 \\
\pm 20 & 50 \\
\pm 10 & 100 \\
0 & 100 \\
\hline
\end{tabular}


Table S3: Computation time for FEP calculations of short-chain alcohols. Each FEP calculation was performed in 20 windows, during which the coupling parameter $\lambda$ was varied from 0 to 1 . We simulated each window for $10 \mathrm{~ns}$, resulting in a total of $200 \mathrm{~ns}$ for the FEP calculation at a given $z$.

\begin{tabular}{c|c}
\hline$z(\AA)$ & Total simulation time (ns) \\
\hline 0 & 200 \\
4 & 200 \\
13 & 200 \\
22 & 200 \\
30 & 200 \\
\hline \multicolumn{2}{c}{ Aggregate simulate time $(\mu \mathrm{s}) 1$} \\
\hline
\end{tabular}



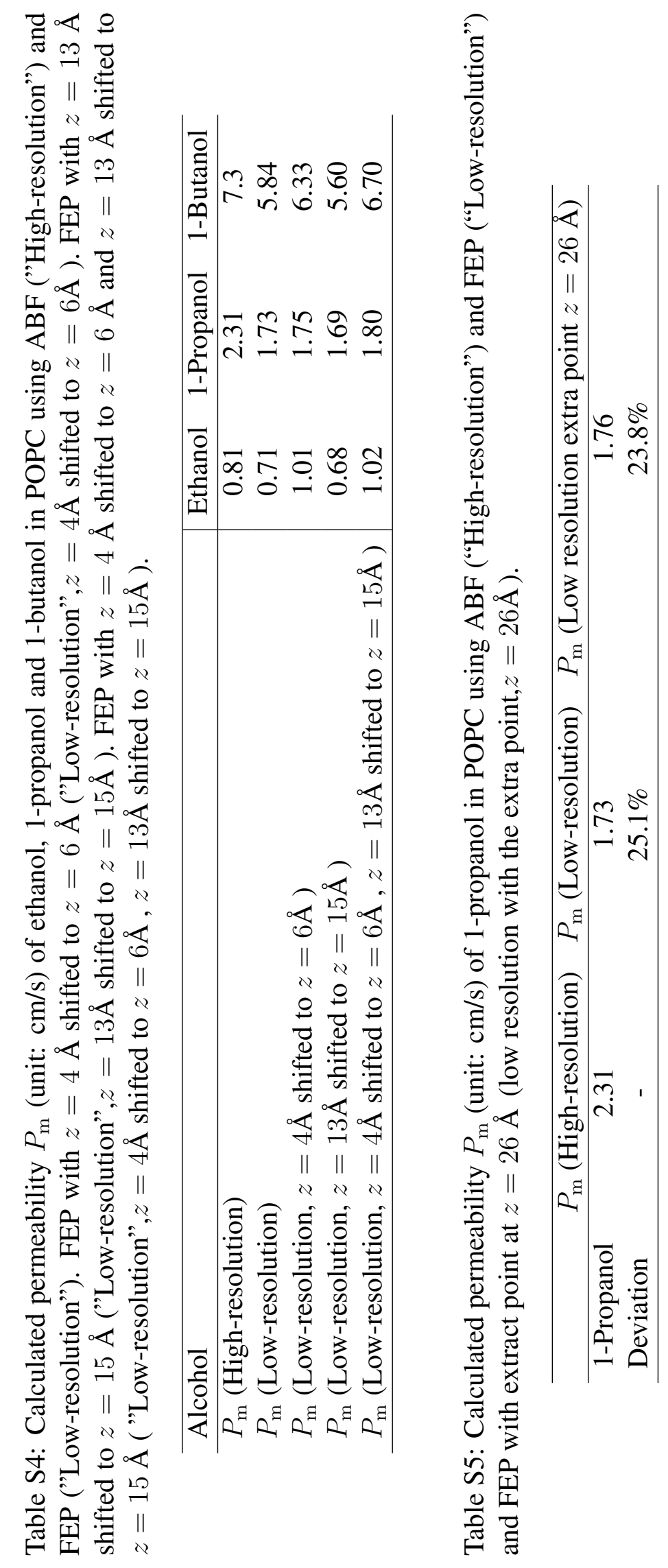

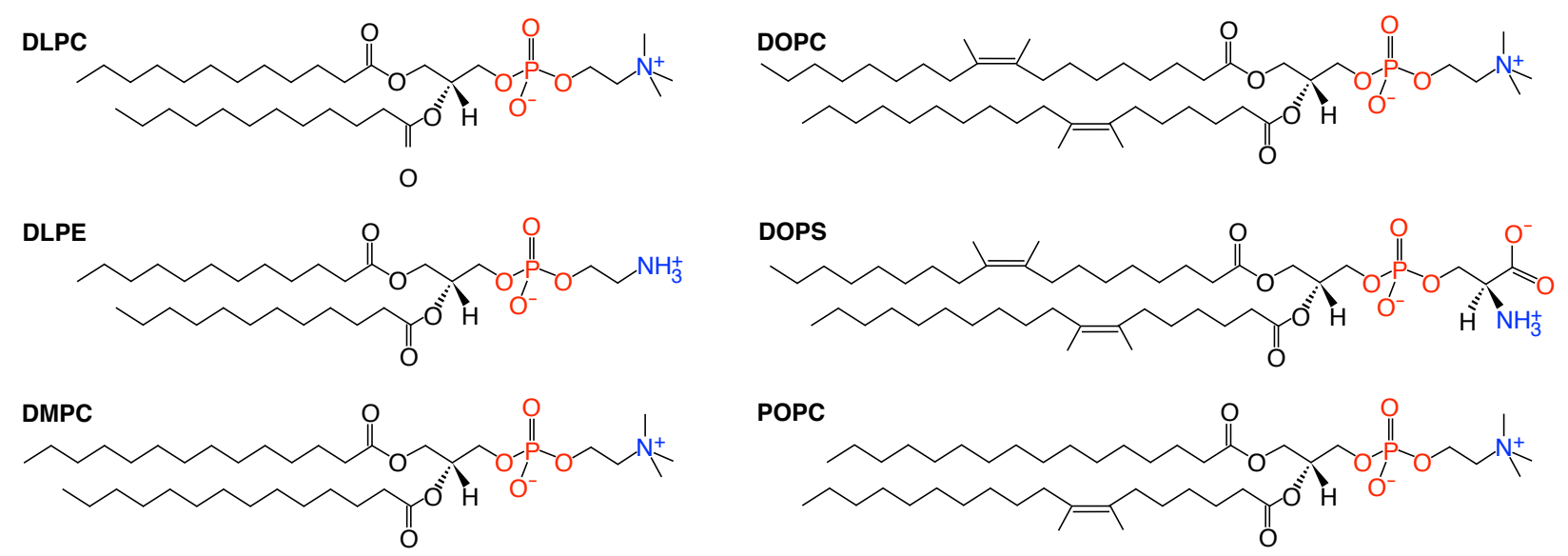

Figure S1: Chemical structures of molecules from six single-species lipid bilayers studied in this work. 

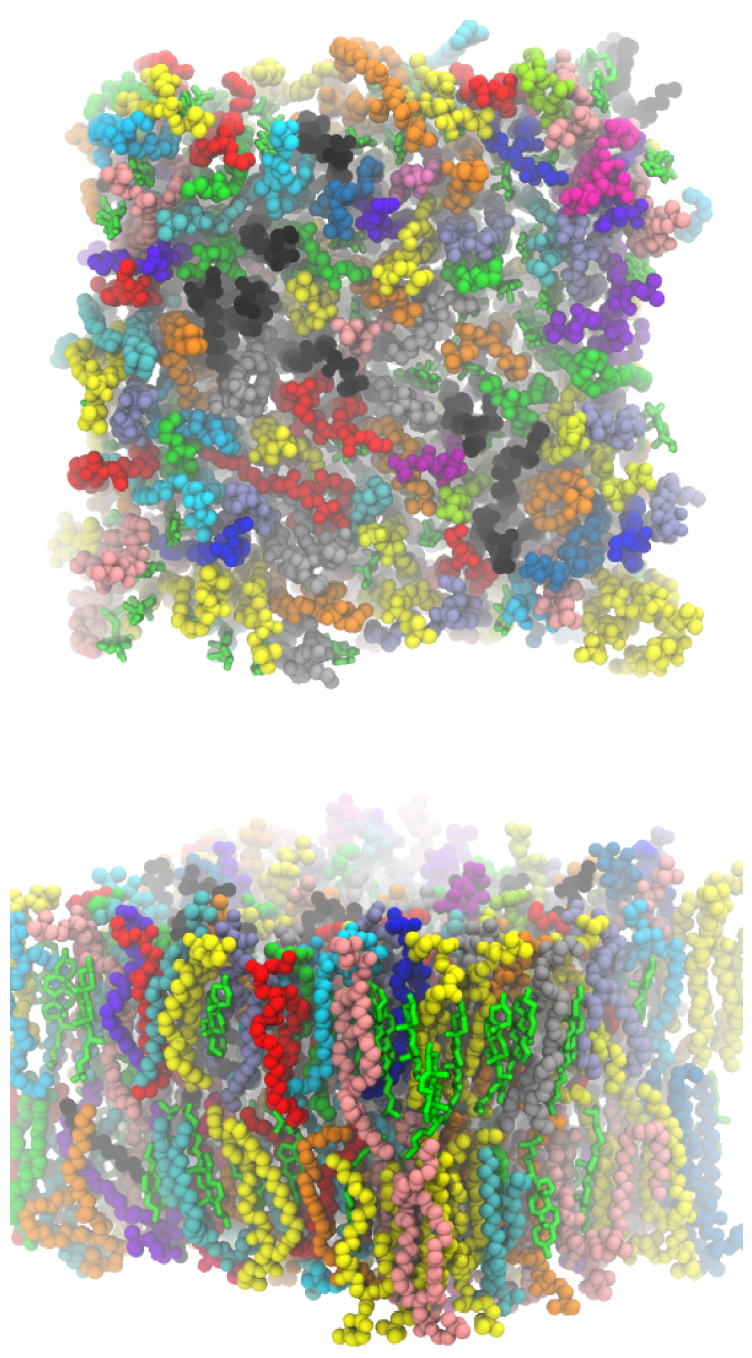

Figure S2: Top (top) and side (bottom) views of the all-atom mammalian membrane model. For clarity, water and ions were not shown. 


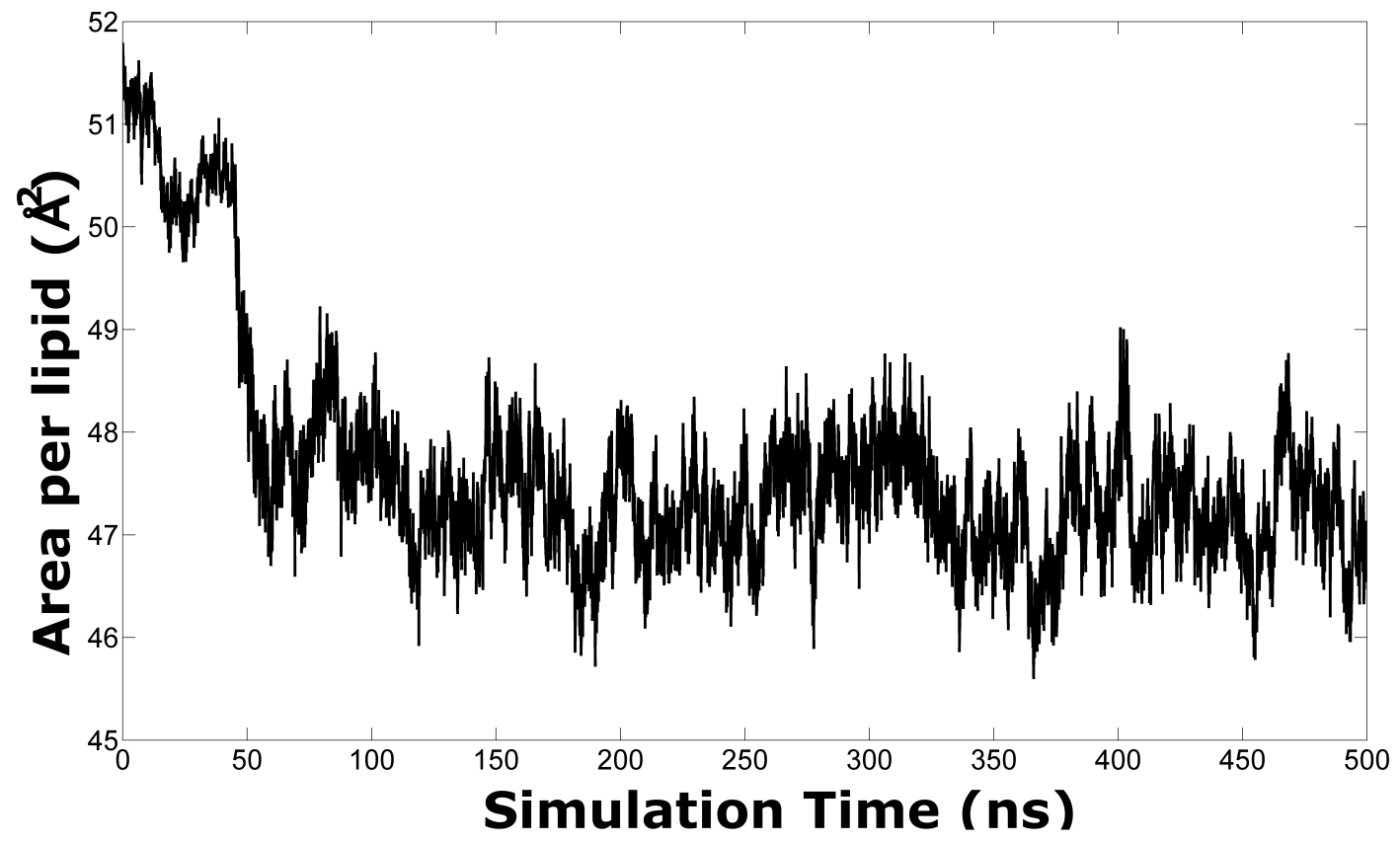

Figure S3: Area per lipid of the mammalian cell membrane model in 500-ns equilibration prior to being used in the $\mathrm{ABF}$ calculations.

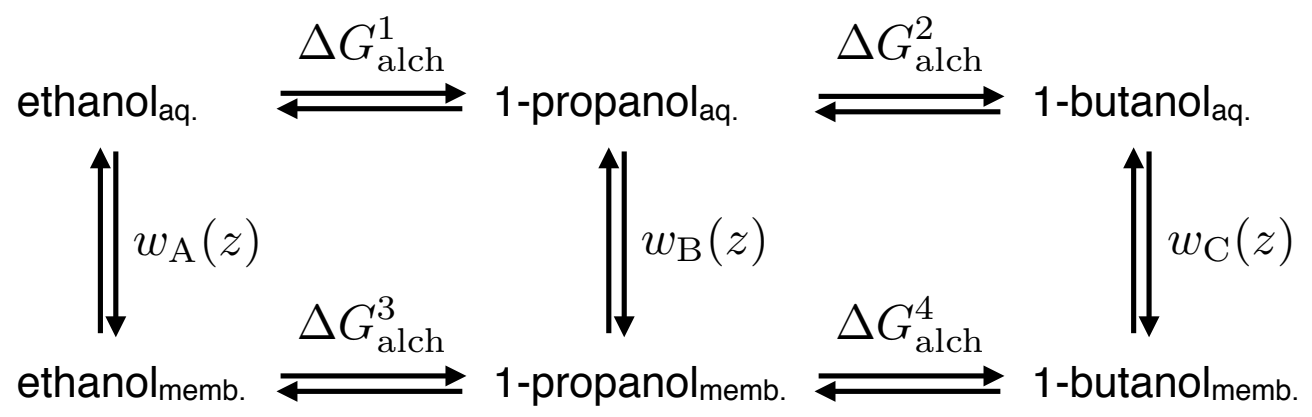

Figure S4: Thermodynamic cycle of short-chain alcohol mutation. The subscripts "aq" and "memb" stand for the aqueous solution and the lipid membrane, respectively. 

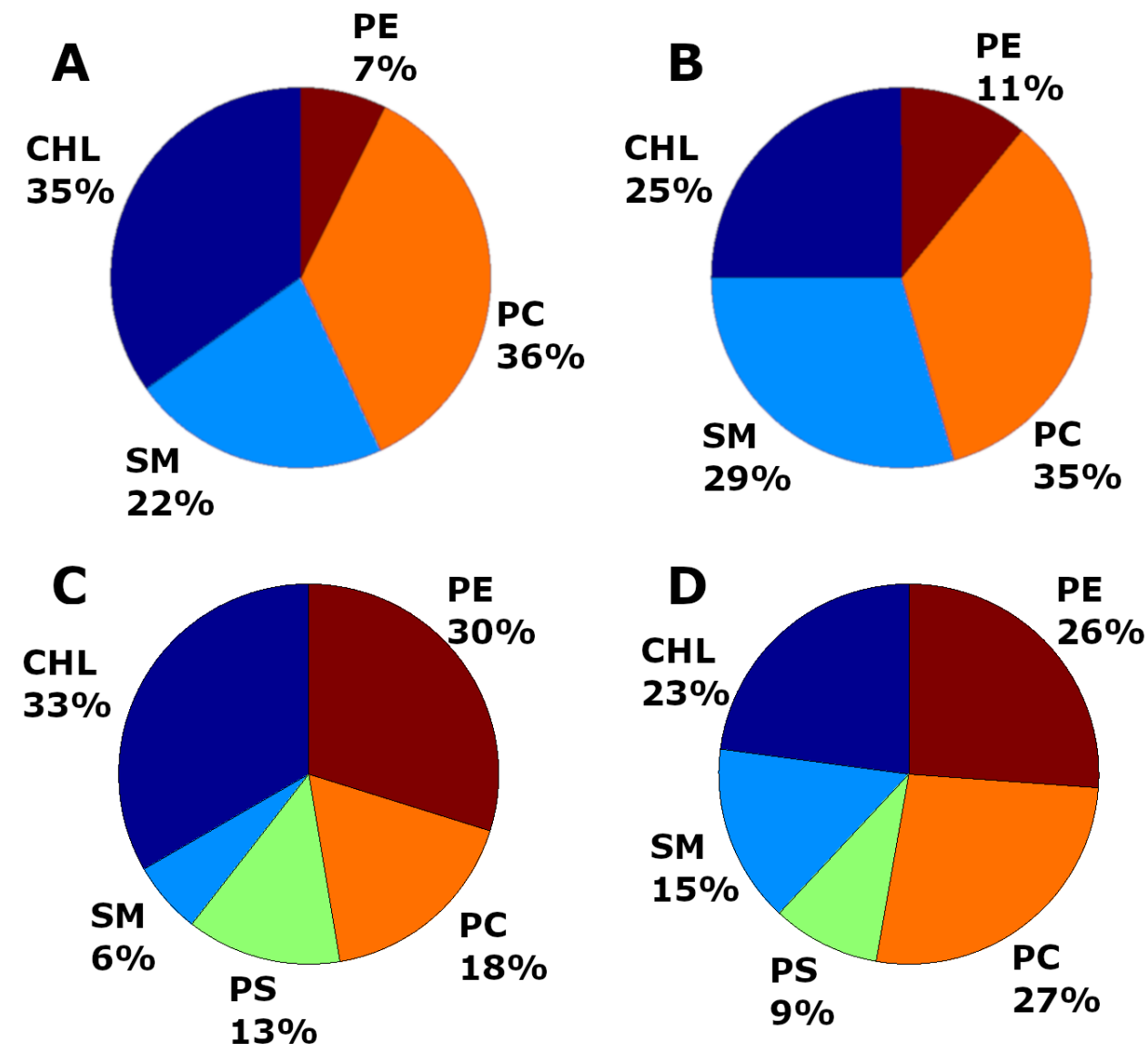

Figure S5: Distribution of lipid species in the outer leaflet (A) and inner leaflet (C) of the mammalian cell membrane model, and distribution of lipid species within $5 \AA$ of the permeant ethanol molecule in the outer leaflet (B) and inner leaflet (D) throughout the ABF simulation. PE: phosphatidylethanolamine, PC: phosphatidylcholine, PS: phosphatidylserine, SM: sphingomyelin , CHL: cholesterol. 


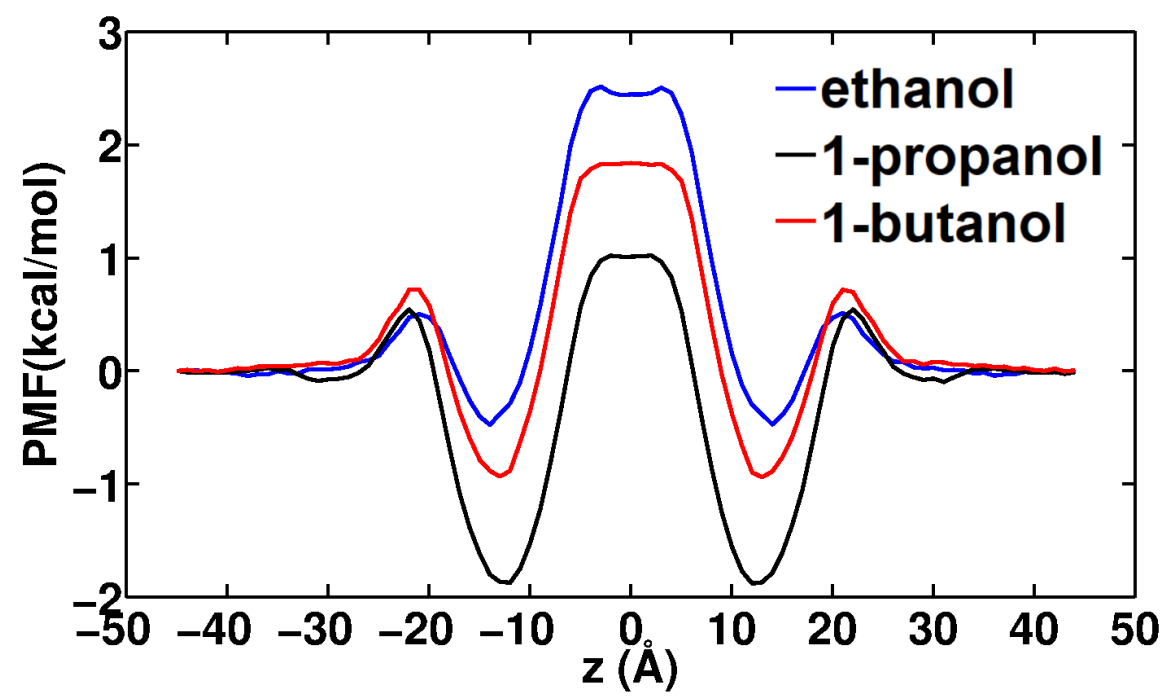

Figure S6: Free-energy profiles $w(z)$ for the permeation of ethanol, 1-propanol and 1-butanol in POPC.
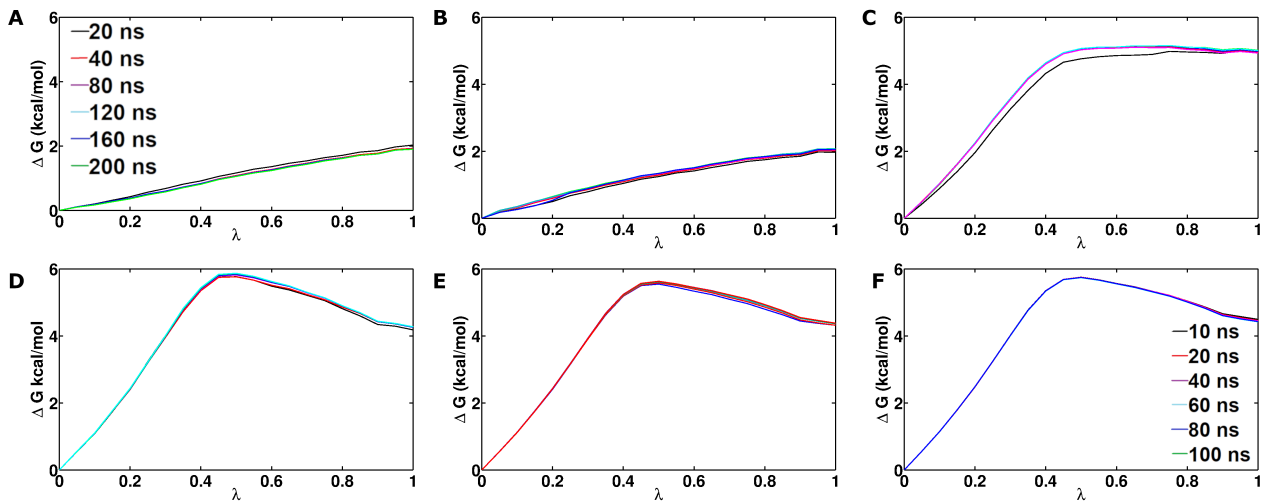

Figure S7: Convergence of FEP calculations indicated by free energy change $(\Delta G)$ of ethanol annihilation in various locations $(z=0 \AA$ (A), $z=4 \AA$ (B), $z=13 \AA$ (C), $z=22 \AA$ (D), $z=30 \AA(\mathrm{E}))$ within the POPC membrane system and in bulk water (F) as the amount of sampling increases. At a given $z$ within the membrane system, FEP was performed in 20 windows with 10-ns sampling in each window. In bulk water, FEP was performed in 20 windows with 5-ns sampling in each window. The sampling amount indicated by the curves represent the total amount of sampling in a FEP calculation, i.e., up to $20 \times 10=200 \mathrm{~ns}$ for a given $z$ location within the membrane system (A-E) and up to $20 \times 5=100 \mathrm{~ns}$ for bulk water $(\mathrm{F})$. 

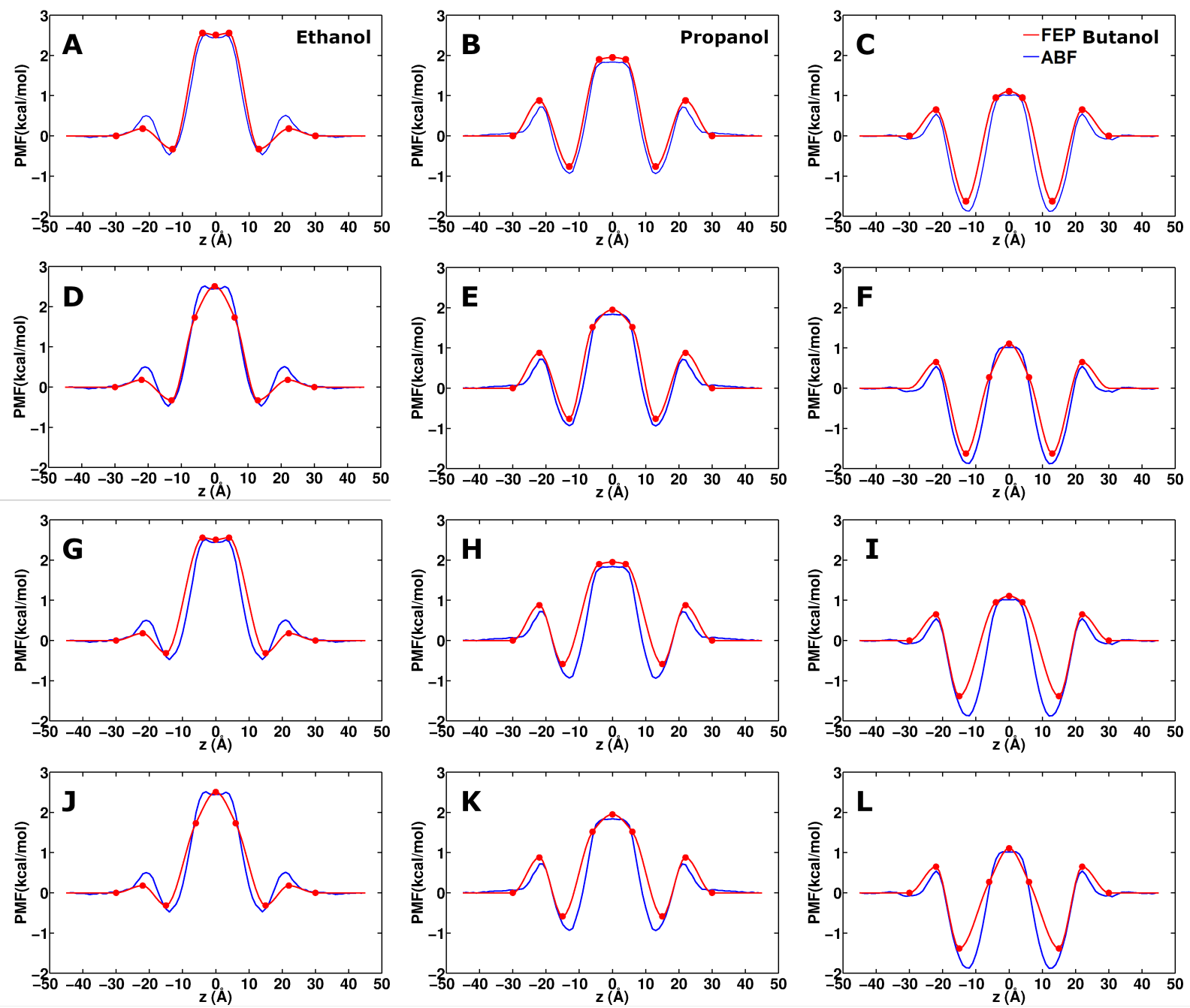

Figure S8: PMFs of ethanol (A,D,G,J), 1-propanol (B,E,H,K) and 1-butanol (C,F,I,L) in POPC. The reference PMFs (blue) were obtained from Comer and Chipot, using the ABF algorithm. The FEP calculations were performed at selected points (red dots) and subsequently used to generate the model PMFs (red) via interpolation. The sampling point at $z=4 \AA$ (A,B,C) was deliberately shifted to $z=6 \AA$ (D,E,F), and that at $z=13 \AA$ (A,B,C) to $z=15 \AA$ (G,H,I). Both sampling points at $z=4 \AA$ and $z=13 \AA$ were shifted, to $z=6 \AA$ and $z=15 \AA$ respectively (J,K,L), to mimic the effect of a "poor guess" on the reproduction of the reference PMF. 

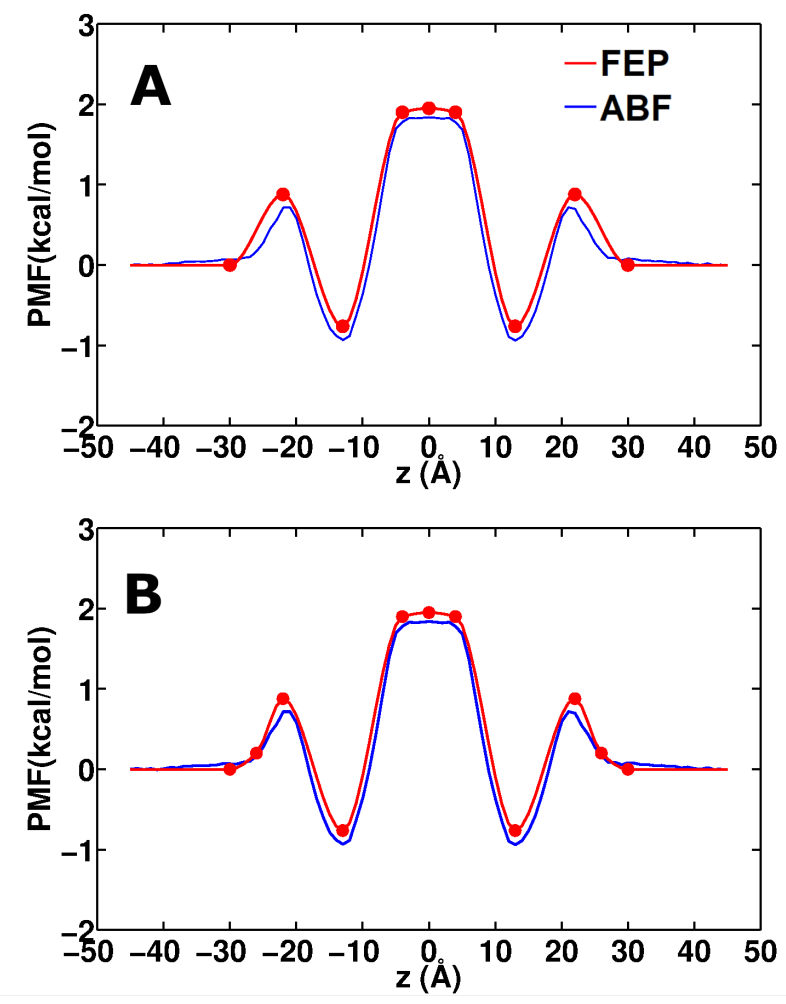

Figure S9: PMFs of 1-propanol in POPC without (A) and with (B) the additional sampling point at $z=26 \AA$. 